\title{
A Study on the Genetic Inheritance of Ankyloglossia Based on Pedigree Analysis
}

\author{
Soo-Hyung Han ${ }^{1}$, Min-Cheol Kim ${ }^{1}$, Yun-Seok Choi ${ }^{2}$, Jin-Soo Lim ${ }^{1}$, Ki-Taik Han ${ }^{1}$ \\ ${ }^{1}$ Department of Plastic and Reconstructive Surgery, St. Vincent's Hospital, Suwon; ${ }^{2}$ Department of Plastic and Reconstructive Surgery, \\ St. Paul's Hospital, Catholic University of Korea School of Medicine, Seoul, Korea
}

Background Ankyloglossia or tongue-tie is a congenital anomaly characterized by an abnormally short lingual frenum. Its prevalence in the newborn population is approximately $4 \%$. Its mode of inheritance has been studied in some articles, but no conclusion has been established. Also, no relevant report has been published in Korea. This study was conducted to elucidate the genetic inheritance of ankyloglossia via pedigree analysis.

Methods In this study, 149 patients with no other congenital anomaly who underwent frenuloplasty between March 2001 and March 2010 were studied. Pedigrees were made via pre- or post-operative history taking, and patients with uncertain histories were excluded. In the patient group that showed a hereditary nature, the male-to-female ratio, inheritance rate, and pattern of inheritance were investigated.

Results One hundred (67.11\%) of the patients were male and 49 (32.89\%) were female (male-female ratio $=2.04: 1)$. Ninety-one $(61.07 \%)$ patients reported no other relative with ankyloglossia, and 58 (38.93\%) patients had a relative with this disease. The inheritance rate was $20.69 \%$ in the 58 cases with a hereditary nature. In the group with no family history of ankyloglossia, the male-female ratio was 3.79:1, which significantly differed from that of the group with a family history of ankyloglossia. X-chromosome mediated inheritance and variation in the gene expression was revealed in the pedigree drawn for the groups with hereditary ankyloglossia.

Conclusions Ankyloglossia has a significant hereditary nature. Our data suggest X-linked inheritance. This study with 149 patients, the first in Korea, showed X-linked inheritance in patients with a sole anomaly.

Keywords Lingual frenum / Pedigree / X-chromosome
Correspondence: Min-Cheol Kim Department of Plastic and

Reconstructive Surgery, St. Vincent's

Hospital, The Catholic University of

Korea School of Medicine,

93 Jungbu-daero, Paldal-gu, Suwon

442-723, Korea

Tel: +82-31-249-8277

Fax: +82-31-241-0005

E-mail: wisethoth@hotmail.com

This article was presented as a poster at the 10th Korea-Japan Congress of Plastic and Reconstructive Surgery on June 16-18, 2010 in Busan, Korea.

No potential conflict of interest relevant to this article was reported.

Received: 30 Mar 2012 • Revised: 4 May 2012 • Accepted: 7 May 2012

pISSN: 2234-6163 • elSSN: 2234-6171 • http://dx.doi.org/10.5999/aps.2012.39.4.329・Arch Plast Surg 2012;39:329-332

\section{INTRODUCTION}

Ankyloglossia is a congenital anomaly in which the lingual frenum is unusually short and thick, thus decreasing lingual mobility $[1,2]$. Its prevalence in the newborn population is about $4 \%$ on average, but varies greatly from $0.02 \%$ to $10.7 \%$ in studies. Its definition and severity classification have not yet been clearly established $[3,4]$. It mostly appears as a sole anomaly, though it is sometimes accompanied by other congenital anomalies such as cleft palate $[3,5]$. As it has a hereditary nature, it occurs more commonly in male children and has been suggested to be related to the X-chromosome [3,6-8]. No local studies have been per- 
formed on the hereditary nature of ankyloglossia in Koreans, and thus, the relevant statistics are all based on international studies.

Thus, in this study, the hereditary nature of ankyloglossia in Koreans was described by identifying and analyzing the pedigrees of patients with ankyloglossia.

\section{METHODS}

This study was performed on 149 patients who underwent frenuloplasty in this hospital within the period of March 2001 to March 2010.

In the screening phase, patients with other anomalies or uncertain family histories were excluded. The pre- and postoperative family histories were taken from the patients' guardians, and the male-female ratio, hereditary rate, and mode of inheritance were calculated in each group with hereditary ankyloglossia.

\section{RESULTS}

At the time of surgery, the mean age of the patients was 3.3 years, and the male-female ratio was 2.04:1 (male: $n=100$ [67.11\%] and female: $\mathrm{n}=49[32.89 \%])$. Fifty-eight $(38.93 \%)$ patients had another family member who had ankyloglossia and thus had

\section{Table 1. Male-female ratio of ankyloglossia}

\begin{tabular}{|lcccc|}
\hline Group & Male & Female & $\begin{array}{c}\text { Male- } \\
\text { Female ratio }\end{array}$ & $\begin{array}{c}\text { P- } \\
\text { value }^{\text {a) }}\end{array}$ \\
\hline Sporadic group $(n=91)$ & $72(79.1)$ & $19(20.9)$ & $3.19: 1$ & 0.0002 \\
Hereditary group $(n=58)$ & $28(48.3)$ & $30(51.7)$ & $0.90: 1$ & \\
\hline Ann & &
\end{tabular}

Among all patients, the ratio was 2.04:1. Male excess was seen only in the sporadic group (i.e, the patient group with no family history of ankyloglossia), which suggested $X$-linked recessive inheritance of tongue-tie, and it was statistically significant $(\mathrm{P}<0.05)$.

Values are presented as number (\%).

a) Tested by chi-square test. hereditary ankyloglossia; 91 (61.07\%) patients had no family member with this anomaly. The male-female ratios in the former and latter groups were 0.90:1 and 3.79:1, respectively, and the proportion of male patients was significantly high only in the sporadic group $(\mathrm{P}=0.0002$, tested by the chi-squared test) (Table 1).

The authors traced the pedigree of the patients who had a family history of ankyloglossia $(n=58)$ and classified them into groups. Group A consisted of patients with relatives with tongue-tie only in the third generation; group $\mathrm{B}$, in the second generation; and group $\mathrm{C}$, in the first generation (Fig. 1). Of the 58 patients with hereditary tongue-tie, 46, 6, and 6 fell under groups $\mathrm{A}, \mathrm{B}$, and $\mathrm{C}$, respectively, and the inheritance rate based on the 12 patients from groups B and C was $20.69 \%$ (95\% confidence interval of observed proportion was $11.17 \%$ to $33.35 \%$ ).

Of the 12 patients who had a parent or grandparent with ankyloglossia as well, nine were male and three were female. Six of the nine male patients had fathers with ankyloglossia, and the remaining three male patients had affected mothers. The three female patients had affected fathers but no affected mothers.

\section{DISCUSSION}

Ankyloglossia is a congenital anomaly that has a high prevalence rate and may cause difficulty in breastfeeding or speech problems for affected infants $[1,2]$. Its treatment options include such surgical procedures as frenotomy, frenectomy, and frenuloplasty, though its spontaneous resolution is also possible in some cases. Currently, various modified surgical procedures such as the use of $\mathrm{a} \mathrm{CO}_{2}$ laser as an auxiliary method are being attempted $[4,5]$. No widely accepted criteria have been established, however, for the surgical indications and the selection of surgical procedures [5]. The exact pathological mechanism of ankyloglossia remains unclear [9], and its conclusively hereditary nature has yet to be elucidated, though numerous relevant

\section{Fig. 1. Patient group with a family history of ankyloglossia $(n=58)$}

Relatives with tongue-tie were seen only in the third generation. (A) Relatives with tongue-tie were seen in the second generation. (B) Relatives with tongue-tie were seen in the first generation. (C) The first generation was omitted in groups A and B, except for one case in group A. The inheritance rate measured in groups B and C among the 58 patients, was 20.69\% (95\% of confidence interval, 11.17\% to 33.35\%). The relevance with the $\mathrm{X}$-chromosome was seen in groups $\mathrm{B}$ and $\mathrm{C}$, and the variation in gene expression in group $\mathrm{A}$ should be considered.

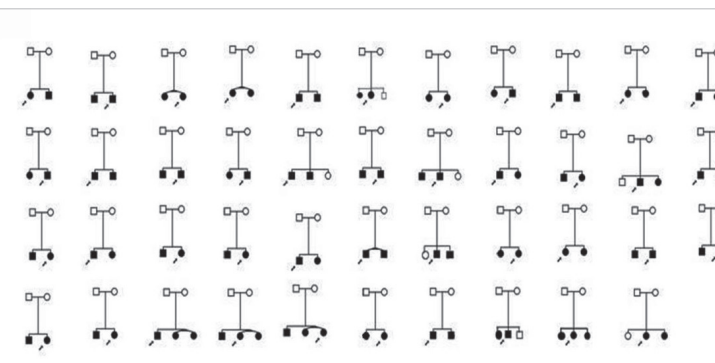


studies have been performed [9]. Ankyloglossia often appears as a single anomaly, though it sometimes occurs as a symptom of such rare syndromes as X-linked cleft palate. A study of the risk factors of ankyloglossia showed that the morbidity among infants whose mothers smoked cocaine was three times that among infants whose mothers did not smoke cocaine [10], and Ballard et al. [4] showed that about $21 \%$ of pediatric patients had affected parents. In addition, based on a pedigree analysis, Klockars reported that ankyloglossia is an autosomal dominant disorder [3,9]. Even though the definition and classification of its severity are inconsistent [3-5], which limits its clinical study, no relevant studies on its hereditary nature have been performed in Korea. As such, in this study, the hereditary nature of ankyloglossia was studied via a basic investigation and analysis of the pedigree of the patients.

Authors showed that the inheritance rate of ankyloglossia was about $20.69 \%$ through the history-taking across the three generations that included the patients themselves. The male-female ratio was 2.04:1, which was similar to the male-female ratio (1.52.6:1) in other studies [1-3], which indicates that the sample of selected patients was appropriate for this study.

The trend towards a higher male proportion was significant only in the group with non-hereditary ankyloglossia. This result is consistent with the assumptions that ankyloglossia is inherited through $\mathrm{X}$-linked recession and that the mothers of the female patients were carriers, and that the patients may have inherited their ankyloglossia. It is also consistent with the results reported by Klockars and Pitkaranta [3].

A correlation with X-linked recession was observed in many patients in groups $\mathrm{B}$ and $\mathrm{C}$ (Fig. 1B, C), wherein the gene was expressed in the parents or grandparents of the patients as shown from the pedigrees traced for the groups with hereditary tonguetie. In the group in which the gene was expressed only in the third generation (Fig. 1A), no clear correlation with the X-chromosome was observed. These findings indicate variations in gene expression, whereby the gene expression follows not only simple principles such as Mendel's Law, but others as well.

The variation in gene expression occurs due to such complex phenomena as genetic features, the interaction among the genes, the biochemical association involved in gene expression, and the environmental conditions in ontogenesis. Particularly, it is well known that non-allelic genes that have different alleles interact.

For example, although genes associated with thalassemia and sickle cell anemia are in different loci, when a patient has heterozygotes of both thalassemia and sickle cell anemia, the heterozygotes synergistically act as homozygotes and cause serious anemia that leads to mortality [11]. In contrast, a human who secretes the $\mathrm{H}$ antigen, a precursor of the $\mathrm{ABO}$ blood group of antigens, into the saliva and other humors is called a secretor, and expresses the dominant gene Se. Thus, the expression can be inhibited in a non-secretor because the $\mathrm{A}$ and $\mathrm{H}$ antigens are not secreted in the saliva even if the blood type of the person is $\mathrm{A}[12]$.

There have been other studies on the correlation of ankyloglossia with the X-chromosome and variations in gene expression, particularly the mutation of the TBX22 gene [6-8]. In patients with X-linked cleft palate, the hereditary trend coincides well with Mendel's laws. In those patients, the mutation of the TBX22 gene was observed and thus, the CPX gene was expressed and ankyloglossia occurred as one of the symptoms of the syndrome. In addition, patients with X-linked cleft palate may present only ankyloglossia or various other physical findings from incomplete to complete cleft palates. This is because mutations can occur not only in the promoter site of the transcription region but also in the promoter site of the non-transcription region in the TBX22 gene, modifying the transcription activity and resulting in various mutations [8]. This study is meaningful because it is the first study in South Korea to show the association between ankyloglossia and the X-chromosome in patients who have no anomaly other than ankyloglossia.

This study has the limitation of being based on a hypothesis of a pattern of inheritance, because gene analysis was not performed. As the exact inheritance mechanism was not identified, some cases were not consistent with the X-linked recessive inheritance, which was one of the inheritance mechanisms we suggested. Additionally, the classification of the groups was challenging. For example, of the patients who belonged to hereditary group $\mathrm{B}$ or $\mathrm{C}$, there were some male patients whose fathers were affected and mothers were unaffected. If the mothers were a carrier (X'X), the inheritance mechanism would be consistent with X-linked recessive inheritance hypothesis, but this cannot be proven. If the mothers were not carriers (XX), the inheritance would have been due to a different mechanism or could have been classified into the sporadic group.

Although the groups should be classified differently to clear confusion in this case, we determined that classification of patients according to a certain unproven mechanism of inheritance was likely to prevent the possibility of identifying other types of inheritance. Therefore, in this study, we presented the groups that were classified based on the criteria of whether the patients had affected relatives nd if so, to which generation the affected relatives belonged. It is expected that the inheritance trend of ankyloglossia will be identified more clearly in gene-level studies on ankyloglossia that will be based on the outcomes of this study.

In this study, the ankyloglossia inheritance trend was investi- 
gated by analyzing the patients' pedigrees. The inheritance rate was $20.69 \%$, and the analysis of the male-to-female ratio and the hereditary trend according to sex showed the possibility of Xlinked inheritance. The inheritance trend revealed by the pedigree showed variations in the gene expression also. This study is of value because it is the first study in South Korea in which a large number of patients who had a single anomaly, ankyloglossia, were investigated.

\section{REFERENCES}

1. Messner AH, Lalakea ML, Aby J, et al. Ankyloglossia: incidence and associated feeding difficulties. Arch Otolaryngol Head Neck Surg 2000;126:36-9.

2. Segal LM, Stephenson R, Dawes M, et al. Prevalence, diagnosis, and treatment of ankyloglossia: methodologic review. Can Fam Physician 2007;53:1027-33.

3. Klockars T, Pitkaranta A. Inheritance of ankyloglossia (tongue-tie). Clin Genet 2009;75:98-9.

4. Ballard JL, Auer CE, Khoury JC. Ankyloglossia: assessment, incidence, and effect of frenuloplasty on the breastfeeding dyad. Pediatrics 2002;110:e63.
5. Suter VG, Bornstein MM. Ankyloglossia: facts and myths in diagnosis and treatment. J Periodontol 2009;80:1204-19.

6. Braybrook C, Doudney K, Marcano AC, et al. The T-box transcription factor gene TBX22 is mutated in X-linked cleft palate and ankyloglossia. Nat Genet 2001;29:179-83.

7. Marcano AC, Doudney K, Braybrook C, et al. TBX22 mutations are a frequent cause of cleft palate. J Med Genet 2004;41:68-74.

8. Pauws E, Moore GE, Stanier P. A functional haplotype variant in the TBX22 promoter is associated with cleft palate and ankyloglossia. J Med Genet 2009;46:555-61.

9. Klockars T. Familial ankyloglossia (tongue-tie). Int J Pediatr Otorhinolaryngol 2007;71:1321-4.

10. Harris EF, Friend GW, Tolley EA. Enhanced prevalence of ankyloglossia with maternal cocaine use. Cleft Palate Craniofac J 1992;29:72-6.

11. Ballas SK. Effect of alpha-globin genotype on the pathophysiology of sickle cell disease. Pediatr Pathol Mol Med 2001;20:107-21.

12. Marcus DM. The ABO, Hh, secretor, and Lewis systems. Immunol Ser 1989;43:685-99. 